\title{
Integrative MicroRNAome Analysis of Skeletal Muscle of Colossoma Macropomum (Tambaqui), Piaractus Mesopotamicus (Pacu), and the Hybrid Tambacu, based on Next-generation Sequencing Data.
}

\section{Bruno EA Fantinatti}

Universidade Estadual Paulista Julio de Mesquita Filho Coordenadoria Geral de Bibliotecas

\section{Erika S Perez}

Universidade Estadual Paulista Julio de Mesquita Filho

\section{Bruna TT Zanella}

Universidade Estadual Paulista Julio de Mesquita Filho

Jéssica S Valente

Universidade Estadual Paulista Julio de Mesquita Filho

Tassiana G de Paula

Universidade Estadual Paulista Julio de Mesquita Filho

\section{Edson A Mareco}

Universidade do Oeste Paulista

Robson F Carvalho

Universidade Estadual Paulista Julio de Mesquita Filho

\section{Silvano Piazza}

Universita degli Studi di Trento

Michela A Denti

Universita degli Studi di Trento

Maeli Dal Pai ( $\square$ maeli.dal-pai@unesp.br)

Universidade Estadual Paulista Júlio de Mesquita Filho https://orcid.org/0000-0001-7269-9197

Research article

Keywords: miRNAoma, skeletal muscle, fish

Posted Date: October 5th, 2020

DOI: https://doi.org/10.21203/rs.3.rs-51788/v1 
License: (c) (i) This work is licensed under a Creative Commons Attribution 4.0 International License. Read Full License

Version of Record: A version of this preprint was published at BMC Genomics on April 6th, 2021. See the published version at https://doi.org/10.1186/s12864-021-07513-5. 


\section{Abstract}

Background: Colossoma macropomum (tambaqui) and Piaractus mesopotamicus (pacu) are good fish species for aquaculture. The tambacu, individuals originating from the induced hybridization of the female tambaqui with the male pacu, present rapid growth and robustness, characteristics which have made the tambacu a good choice for Brazilian fish farms. Here, we used small RNA sequencing to examine global miRNA expression in the genotypes pacu (PC), tambaqui (TQ), and hybrid tambacu (TC), (Juveniles, $n=5$ per genotype), in order to better understand the relationship between tambacu and its parental species, and also to clarify the mechanisms involved in tambacu muscle growth and maintenance based on miRNAs expression.

Results: Regarding differentially expressed (DE) miRNAs between the three genotypes, we observed 8 upregulated and 7 downregulated miRNAs considering TC vs. PC; 14 miRNAs were upregulated and 10 were downregulated considering TC vs. TQ, and 15 miRNAs upregulated and 9 were downregulated considering PC vs. TQ. The majority of the miRNAs showed specific regulation for each genotype pair, and no miRNA were shared between the 3 genotype pairs, in both up- and down-regulated miRNAs. Considering only the miRNAs with validated target genes, we observed the miRNAs miR-144-3p, miR-1385p, miR-206-3p, and miR-499-5p. GO enrichment analysis showed that the main target genes for these miRNAs were grouped in pathways related to oxygen homeostasis, blood vessel modulation, and oxidative metabolism.

Conclusions: Our global miRNA analysis provided interesting DE miRNAs in the skeletal muscle of pacu, tambaqui, and the hybrid tambacu. In addition, in the hybrid tambacu, we identified some miRNAs controlling important molecular muscle markers that could be relevant for the farming maximization.

\section{Background}

Colossoma macropomum (tambaqui) and Piaractus mesopotamicus (pacu) are fish species that have widely accepted in the consumer market. In addition, they present desirable characteristics for an intensive breeding environment as rapid growth and optimal adaptation to artificial feeding [1].

It is known that Hybrid individuals possess desirable characteristics for production, such as high growth rate, higher resistance to disease, and higher quality of meat [2]. For this reason, several producers have chosen to cultivate the hybrid between the induced crossing of the tambaqui female and the pacu male, the tambacu. Tambacu, in spite of the few genetic information, has the capacity to be more resistant to parasites and stress [3]. Furthermore, tambacu is considered a fish with great potential for Brazilian aquaculture, since it presents a high and fast growth rate, including the skeletal muscle and higher resistance to low temperatures, which contribute to increasing their rusticity [4], thus representing an important model to study. The skeletal muscle is directly involved in the growth of the fish, corresponding to about $35-60 \%$ of the body weight of the animal [5]. This abundant muscle mass enables the survival of 
the animals in the aquatic environment and is commercially important for animal production, since it is one of the most important food sources for human diet [6].

With the focus of massive data analysis, non-coding RNAs have been highly explored in recent years considering their strong impact on controlling several biological processes [7], [8]. Among non-coding RNAs, miRNAs (miRNAs), a well reported class of small non-coding RNAs, are known to perform a fine regulation of gene activity in a post-transcriptional mode upon their association with RNA-induced silencing complex (RISC) and binding via base-complementarity to target mRNAs, resulting in their translational repression and/or in their degradation [9], [10].

Some miRNAs are known to be specifically or highly expressed in the cardiac and/or skeletal muscles and have been dubbed "myo-miRs" [11]. In fish, recent studies have brought information regarding the evolution and genomic organization of myo-miRNAs [12].

Considering the important role of miRNAs in cell physiology, we investigated muscle miRNA profiles as a powerful approach to study the relationship between tambacu and its parental genotypes, to better understand the factors involved in tambacu muscle development, growth, and maintenance. In this way, the present work can be an important source of information and supports studies that address the advantages of adopting hybrids for cultivation, which are still scarce [4].

\section{Results}

Early juvenile fish Colossoma macropomum, Piaractus mesopotamicus, and hybrid tambacu were studied. All the extracted RNA samples were analyzed by NanoVue (GE Healthcare), and Bioanalyzer (Agilent Technologies) and only samples with a RIN $\geq 8$ were selected for the sequencing procedures.

After preprocessing steps, the number of reads decreased to approximately $50 \%$ due to the removal of low-quality reads and the adapters, and the removal of sequences not matching the minimum/maximum size corresponding to miRNAs (Fig.1, Table 1, and Additional file 1). We obtained a total alignment average of $51,78 \%$, considering all samples analyzed. As a result of featureCounts processing, a count matrix was generated for each miRNA present in the reference (see Additional file 2). As part of the pipeline, Principal Component Analysis (PCA) (Fig. 2a) and Dispersion Analysis (Fig. 2b) were run in order to cluster the samples based on the expression values and to observe the quality of the data. We observed the separation clusters among samples, indicating that the different groups correspond to different genotypes. Also, the Dispersion Analysis showed that below a mean read count of 10 , the dispersion of the data increased dramatically. Thus, miRNAs presenting a mean read count $<10$ were filtered out in order to keep only miRNAs not presenting high dispersion levels. 
Table 1

Total read numbers throughout the filtering process. Raw (raw data after downloading), Clipped (remaining reads after removing adaptors and reads shorter than 17nt), Filtered (remaining reads after filtering by read quality) and Sized (remaining reads after removing sequences longer than $26 \mathrm{nt}$ ).

\begin{tabular}{|llllll|}
\hline Samples & Counts & & & & \multicolumn{2}{l}{$\begin{array}{l}\text { Overall Remaining } \\
\text { (\%) }\end{array}$} \\
\cline { 2 - 6 } & Raw & Clipped & Filtered & Sized & 52,52 \\
\hline PC & 57376715 & 42480101 & 39296309 & 30132774 & 52,82 \\
\hline TC & 55492134 & 42720612 & 39513638 & 29310231 & 50,02 \\
\hline TQ & 48058874 & 36611767 & 33906594 & 24038384 & 50,0 \\
\hline
\end{tabular}

Differentially expressed (DE) miRNAs groups were observed in a row-clustered heatmap containing all the comparison genotype pairs (TC vs. PC, TC vs. TQ, and PC vs. TQ) and all the miRNAs detected as DE (Fig. $3)$. We observed the grouping of the DE miRNAs between the samples and genotypes and their characteristics in terms of expression level, according to the color key histogram. Such miRNAs were also observed through non-clustered heat maps, where it was detected the amount of DE miRNAs present in each comparing pair separately, with miRNAs organized in a decreasing log2FC order (Fig. 4).

In order to observe the number of DE miRNAs found regarding Log2FC and the adjusted p-value (padj), we created volcano plots (Fig. 5) showing DE miRNAs in red.

DE analysis showed 8 upregulated and 7 downregulated miRNAs for TC vs. PC genotype pair (Fig. 4a and Fig. 4d, Table 2 and Additional file 3), 14 upregulated and 10 downregulated miRNAs for TC vs. TQ genotype pair (Fig. 4b and Fig. 4e, Table 3 and Additional file 4), and 15 upregulated and 9 downregulated miRNAs for the PC vs. TQ genotype pair (Fig. 4c and Fig. 4f, Table 4 and Additional file 5). 
Table 2

DE miRNAs (TC vs. PC). Up-regulated and down-regulated miRNAs are in bold and italic, respectively, followed by log2FC and padj values. miRNAs with experimentally validated interactions are underlined.

\begin{tabular}{|lll|}
\hline miRNA (TC vs PC) & log2FoldChange & padj \\
\hline dre-miR-216b.path1 & $\mathbf{1 . 9 5 0 1 1 4 6 5 5 0 2 6 2 3}$ & $\mathbf{5 , 7 5 E + 0 7}$ \\
\hline dre-miR-216a.path1 & $\mathbf{1 . 5 2 6 2 2 1 2 8 3 0 6 1 5 5}$ & $\mathbf{5 , 7 5 E + 0 7}$ \\
\hline dre-miR-2188-5p.path1 & $\mathbf{1 . 1 6 3 6 7 9 2 3 0 5 3 0 1 5}$ & $\mathbf{0 . 0 0 7 6 8 6 8 0 1 2 1 7 4 5 8 9 6}$ \\
\hline dre-miR-451.path1 & $\mathbf{1 . 0 1 6 1 5 1 0 9 2 2 7 5 3 7}$ & $\mathbf{0 . 0 2 3 9 6 8 8 6 4 7 0 3 1 3 5 3}$ \\
\hline dre-miR-144-3p.path1 & $\underline{\mathbf{1 . 0 0 3 5 4 4 1 2 3 4 8 8 3 2}}$ & $\underline{\mathbf{0 . 0 2 3 8 8 4 6 4 5 5 0 7 6 9 7}}$ \\
\hline dre-miR-10a-5p.path1 & $\mathbf{0 . 9 9 0 7 7 7 1 0 4 7 0 4 7 0 9}$ & $\mathbf{0 . 0 2 6 4 3 9 7 6 7 5 3 5 4 5 1 2}$ \\
\hline dre-miR-1388-5p.path1 & $\mathbf{0 . 7 9 9 3 5 6 5 4 2 9 9 7 5 8 7}$ & $\mathbf{0 . 0 0 5 9 9 0 2 6 3 4 8 2 9 4 4 3 3}$ \\
\hline dre-miR-210-5p.path1 & $\mathbf{0 . 7 8 8 2 7 8 4 8 9 0 5 2 1 2 5}$ & $\mathbf{0 . 0 0 1 0 1 2 3 6 4 6 5 3 7 3 1 8 5}$ \\
\hline dre-miR-22a-5p.path1 & -0.757442401072745 & 0.000145626758222292 \\
\hline dre-miR-138-5p.path1 & $\underline{-0.785285789697823}$ & $\underline{0.045680698066672}$ \\
\hline dre-miR-199-3-3p.path1 & -0.805140039326324 & 0.000392850813725473 \\
\hline dre-miR-375.path2 & -0.814180847823428 & 0.0496601836495912 \\
\hline dre-miR-375.path1 & -0.824148790777728 & 0.0467129686944908 \\
\hline dre-miR-132-3p.path2 & -0.85811978487659 & 0.0212262367860986 \\
\hline dre-miR-132-3p.path1 & -0.860515272540314 & 0.0212262367860986 \\
\hline
\end{tabular}


Table 3

DE miRNAs (TC vs. TQ). Up-regulated and down-regulated miRNAs are in bold and italic, respectively, followed by log2FC and padj values. miRNAs with experimentally validated interactions are underlined.

\begin{tabular}{|lll|}
\hline miRNA (TC vs TQ) & log2FoldChange & padj \\
\hline dre-miR-499-5p.path1 & $\underline{\mathbf{1 . 4 9 5 8 0 9 9 4 4 2 5 5 9 8}}$ & $\underline{\mathbf{0 . 0 0 0 1 7 3 8 3 9 4 8 1 1 2 7 4 3 9}}$ \\
\hline dre-miR-499-3p.path1 & $\mathbf{1 . 2 3 3 5 6 8 1 2 7 6 5 2 2 9}$ & $\mathbf{0 . 0 0 2 8 2 9 4 6 1 5 6 8 0 6 0 8 1}$ \\
\hline dre-miR-144-3p.path1 & $\underline{\mathbf{1 . 1 6 6 5 9 1 3 7 5 0 9 1 4 7}}$ & $\underline{\mathbf{0 . 0 0 4 7 4 9 4 1 0 7 4 2 4 1 2 6 6}}$ \\
\hline dre-miR-122.path1 & $\mathbf{1 . 0 7 6 9 4 1 1 1 0 8 4 9 5 3}$ & $\mathbf{0 . 0 0 2 8 2 9 4 6 1 5 6 8 0 6 0 8 1}$ \\
\hline dre-miR-7a.path3 & $\mathbf{1 . 0 4 9 8 4 4 0 6 4 2 5 9 8 5}$ & $\mathbf{0 . 0 2 9 0 6 5 2 2 7 3 9 8 1 7 4 8}$ \\
\hline dre-miR-184.path1 & $\mathbf{0 . 9 6 7 5 5 7 3 6 0 1 4 4 7 7 1}$ & $\mathbf{0 . 0 1 5 2 0 5 0 5 4 2 4 5 8 1 9 5}$ \\
\hline dre-miR-184.path2 & $\mathbf{0 . 9 6 7 5 5 7 3 6 0 1 4 4 7 7 1}$ & $\mathbf{0 . 0 1 5 2 0 5 0 5 4 2 4 5 8 1 9 5}$ \\
\hline dre-miR-206-3p.path2 & $\underline{\mathbf{0 . 9 3 0 8 0 1 8 1 5 7 7 3 0 2 5}}$ & $\mathbf{0 . 0 0 9 4 7 5 7 2 9 2 1 5 5 1 3 4}$ \\
\hline dre-miR-10a-5p.path1 & $\mathbf{0 . 8 5 7 5 0 5 0 8 7 0 1 8 2 5 5}$ & $\mathbf{0 . 0 4 9 9 6 7 6 7 6 6 8 2 6 8 8 8}$ \\
\hline dre-miR-27d.path1 & $\mathbf{0 . 8 1 6 1 0 5 7 8 6 8 9 2 2 9 1}$ & $\mathbf{3 , 3 6 E + 0 9}$ \\
\hline dre-miR-192.path1 & $\mathbf{0 . 8 0 0 3 3 2 5 2 6 5 3 9 8 4 2}$ & $\mathbf{0 . 0 0 2 8 2 9 4 6 1 5 6 8 0 6 0 8 1}$ \\
\hline dre-miR-27b-3p.path1 & $\mathbf{0 . 7 9 6 9 4 2 1 4 1 2 6 4 3 8 2}$ & $\mathbf{0 . 0 0 0 2 8 7 4 4 5 3 3 0 0 0 3 9 9 1}$ \\
\hline dre-miR-363-3p.path1 & $\mathbf{0 . 7 8 3 9 1 1 5 4 6 0 0 5 4 1 8}$ & $\mathbf{0 . 0 0 0 1 7 3 8 3 9 4 8 1 1 2 7 4 3 9}$ \\
\hline dre-miR-212-5p.path1 & $\mathbf{0 . 7 5 8 0 8 3 0 1 3 6 1 5 1 9 9}$ & $\mathbf{0 . 0 4 8 1 7 6 8 5 0 5 2 4 7 1 2 2}$ \\
\hline dre-miR-187.path1 & -0.771077479498364 & 0.0036724290357109 \\
\hline dre-miR-18a.path1 & -0.772496606919669 & 0.00861750612968111 \\
\hline dre-miR-199-3-3p.path1 & -0.776134338005323 & 0.000598753742363573 \\
\hline dre-miR-29a.path1 & -0.788357562619249 & 0.00295632195436478 \\
\hline dre-miR-221-3p.path1 & -0.810494830489444 & $2,34 E+06$ \\
\hline dre-miR-190a.path1 & -0.815779490936263 & 0.0193213894766877 \\
\hline dre-miR-727-5p.path1 & -0.847542177958638 & 0.0147146666881142 \\
\hline dre-miR-19d-3p.path1 & -0.928688683649729 & 0.000349643237371769 \\
\hline dre-miR-223.path1 & -1.00025248763242 & 0.000674009078964609 \\
\hline dre-miR-155.path1 & -1.0467224627299 & 0.00608254996948173 \\
\hline
\end{tabular}


Table 4

DE miRNAs (PC vs. TQ). Up-regulated and down-regulated miRNAs are in bold and italic, respectively, followed by log2FC and padj values. miRNAs with experimentally validated interactions are underlined.

\begin{tabular}{|lll|}
\hline miRNA (PC vs TQ) & log2FoldChange & padj \\
\hline dre-miR-132-3p.path2 & $\mathbf{1 . 4 4 0 5 1 4 8 9 7 8 2 1 4 7}$ & $\mathbf{2 , 4 9 E + 0 8}$ \\
\hline dre-miR-132-3p.path1 & $\mathbf{1 . 4 4 0 0 2 7 0 8 5 0 7 7 6 6}$ & $\mathbf{2 , 4 9 E + 0 8}$ \\
\hline dre-miR-499-5p.path1 & $\underline{\mathbf{1 . 3 1 8 2 8 2 9 5 3 0 7 6 1 6}}$ & $\mathbf{0 . 0 0 0 9 8 9 1 9 1 5 7 2 8 5 1 5 9 6}$ \\
\hline dre-miR-7a.path3 & $\mathbf{1 . 2 7 6 7 8 0 9 5 6 9 5 1 2 5}$ & $\mathbf{0 . 0 1 0 0 7 1 6 0 5 6 6 4 0 7 6 5}$ \\
\hline dre-miR-499-3p.path1 & $\mathbf{1 . 1 9 8 5 0 8 0 3 2 1 9 8 9 1}$ & $\mathbf{0 . 0 0 4 8 8 5 2 6 0 0 6 5 8 0 0 5 4}$ \\
\hline dre-miR-489.path1 & $\mathbf{1 . 1 3 6 2 3 6 6 1 1 0 5 8 1}$ & $\mathbf{2 , 0 6 \mathrm { E } + 0 4}$ \\
\hline dre-miR-122.path1 & $\mathbf{1 . 0 3 8 6 4 0 2 6 0 8 9 6 2 6}$ & $\mathbf{0 . 0 0 4 8 8 5 2 6 0 0 6 5 8 0 0 5 4}$ \\
\hline dre-miR-146b.path1 & $\mathbf{1 . 0 0 5 9 8 2 7 0 7 1 2 0 0 2}$ & $\mathbf{0 . 0 0 5 7 7 8 9 4 7 4 5 9 8 9 9 8 6}$ \\
\hline dre-miR-26a-2-3p.path1 & $\mathbf{1 . 0 0 0 5 2 7 1 9 7 5 7 4 7 2}$ & $\mathbf{0 . 0 0 1 9 4 4 0 8 8 8 1 9 9 5 7 0 6}$ \\
\hline dre-miR-27d.path1 & $\mathbf{0 . 8 8 1 7 2 5 0 8 0 2 5 3 3 4 5}$ & $\mathbf{1 , 0 0 E + 0 9}$ \\
\hline dre-miR-192.path1 & $\mathbf{0 . 8 7 4 5 6 0 9 1 5 9 5 6 6}$ & $\mathbf{0 . 0 0 0 9 8 9 1 9 1 5 7 2 8 5 1 5 9 6}$ \\
\hline dre-miR-184.path1 & $\mathbf{0 . 8 6 7 8 2 1 4 5 1 8 8 3 6 6 5}$ & $\mathbf{0 . 0 4 1 4 9 1 6 2 6 1 0 1 7 6 7 7}$ \\
\hline dre-miR-184.path2 & $\mathbf{0 . 8 6 7 8 2 1 4 5 1 8 8 3 6 6 5}$ & $\mathbf{0 . 0 4 1 4 9 1 6 2 6 1 0 1 7 6 7 7}$ \\
\hline dre-miR-212-5p.path2 & $\mathbf{0 . 8 3 0 9 2 9 7 8 3 2 6 8 0 4}$ & $\mathbf{0 . 0 4 5 0 4 9 8 5 1 3 1 9 0 5 7 9}$ \\
\hline dre-miR-212-5p.path1 & $\mathbf{0 . 8 1 7 0 7 6 5 7 2 3 0 1 2 3 9}$ & $\mathbf{0 . 0 4 1 4 9 1 6 2 6 1 0 1 7 6 7 7}$ \\
\hline dre-miR-155.path1 & -0.808575426957622 & 0.0483381089257489 \\
\hline dre-miR-19d-3p.path1 & -0.886901002648801 & 0.000711319019021908 \\
\hline dre-miR-223.path1 & -0.918993216115796 & 0.00229667926127593 \\
\hline dre-miR-210-5p.path1 & -1.01067899424562 & $1,48 E+09$ \\
\hline dre-miR-2188-5p.path1 & -1.01503627156949 & 0.0258798964614143 \\
\hline dre-miR-451.path1 & -1.17281189217678 & 0.00812402676379883 \\
\hline dre-miR-216a.path1 & -1.19826926904911 & 0.000237764301717364 \\
\hline dre-miR-2188-3p.path1 & -1.45957650464932 & 0.000407849519588126 \\
\hline dre-miR-216b.path1 & -1.61565155335869 & $9,71 E+09$ \\
\hline
\end{tabular}


According to the Venn diagram data (Figs. 6 and 7), we analyzed the miRNA distribution pattern between the parental species and the hybrid, and it was possible to observe some DE miRNA-sharing between the different genotype's pairs. For example, considering the upregulated set of miRNAs, miR-144-3p and miR10a-5p appeared to be upregulated in TC compared to both PC and TQ (Figs. 6 and 8). This leads us to think that the upregulation of these miRNAs could be a result of a multifactorial characteristic involving both profiles given that such miRNAs are upregulated only when the contrast involves the hybrid and such miRNAs are not DE between parental species. A similar characteristic was observed in the downregulated miRNAs, where miR-199-3-3p appears downregulated in TC when compared with both PC and TQ. These miRNAs are considered as TC-exclusive (in terms of expression levels), as they appear to be up- or down-regulated only in the hybrid compared to the parental species, but not between parental species (Fig. 8). Also, we detected some up- or down-regulated miRNAs only in the hybrid, representing a specific characteristic of this genotype.

We then assigned different miRNAs in 6 different categories regarding inheritance characteristics related to expression levels, i.e., (i) TC-exclusive (for those miRNAs differentially expressed only in TC compared with both parental species), (ii) PC-inherited (for those miRNAs with expression patterns similar to PC expression levels), (iii) TQ-inherited (for those miRNAs with expression patterns similar to TQ expression levels), (iv) TC vs. PC-exclusive (for miRNAs that are differentially expressed only when TC and PC are involved in comparisons), (v) PC vs. TQ-exclusive (for miRNAs that are differentially expressed only when PC and TQ are involved in comparisons), and (vi) TC vs. TQ-exclusive (for miRNAs that are differentially expressed only when TC and TQ are involved in comparisons). miRNAs are also discriminated in up- ( $(\mathbf{A})$ and downregulated ( $\boldsymbol{\nabla})$ (Fig. 8).

In the search for target genes involved in DE miRNAs expression, we searched for experimentally validated targets using miRTarBase dataset version 7 [13]. Two networks comprising only validated data were detected (Fig. 9 and 10). A network presenting strong validated interactions between target genes and DE miRNAs expression for Homo sapiens, Mus musculus, Rattus norvegicus, and Danio rerio are presented (Fig. 9). We observed interactions among 31 known miRNAs, and the top five miRNAs with a higher number of interactions were hsa-miR-221-3p, has-miR-27b-3p, hsa-miR-138-5p, mmu-miR-206-3p, and hsa-miR-132-3p, targeting respectively $72,52,47,32$, and 32 target genes. Considering the Danio rerio validated interaction data (Fig. 10), we observed that the network presents interactions involving four DE miRNAs and 10 target genes: dre-miR-138-5p (vcana), dre-miR-206-3p (vegfa and jun), dre-miR-499-5p (cyb561d2), and dre-miR-144-3p (Imo2, klfd, gata2a, meis1, klf3, and alas2).

Ontology analysis carried out by using Enrichr [14], considering the genes validated as targets of the DE miRNAs (considering the Danio rerio validated interactions) showed that the interacting-validated genes are involved in various categories of ontology. For Biological Processes, Cellular Components, and Molecular Functions, the top enriched terms were, respectively, oxygen homeostasis (the most representative gene was 5 -aminolevulinate synthase 2 - alas2), nuclear euchromatin (the most representative gene was jun), and transcriptional repressor activity, RNA polymerase II activating transcription factor binding (the most representative genes were jun and Imo2) (Figs. 11-13 and Table 5). 
Table 5

Ontology analysis by gene-term enrichment, considering the Danio rerio interacting-validated genes targets of the DE miRNAs, showing the genes and the ontology categories of signaling pathways. alas2 5-aminolevulinate synthase 2; Imo2 - LIM Domain Only 2; jun - AP-1 Transcription factor Subunit; meis1 Meis Homeobox 1.

\begin{tabular}{|c|c|c|}
\hline & Signaling Pathways & Genes \\
\hline \multirow{10}{*}{$\begin{array}{l}\text { Biological } \\
\text { Process }\end{array}$} & oxygen homeostasis (G0:0032364) & alas2 \\
\hline & gas homeostasis (G0:0033483) & alas2 \\
\hline & cellular response to thyroid hormone stimulus (G0:0097067) & Imo2 \\
\hline & $\begin{array}{l}\text { negative regulation of transcription from RNA polymerase II promoter in } \\
\text { response to stress (GO:0097201) }\end{array}$ & jun \\
\hline & regulation of DNA-templated transcription, initiation (G0:2000142) & jun \\
\hline & mRNA transcription from RNA polymerase II promoter (G0:0042789) & Imo2 \\
\hline & negative regulation of hemopoiesis (G0:1903707) & meis1 \\
\hline & negative regulation by host of viral transcription (G0:0043922) & jun \\
\hline & mRNA transcription (G0:0009299) & Imo2 \\
\hline & $\begin{array}{l}\text { positive regulation of vascular smooth muscle cell proliferation } \\
\text { (GO:1904707) }\end{array}$ & jun \\
\hline \multirow{4}{*}{$\begin{array}{l}\text { Cellular } \\
\text { Component }\end{array}$} & nuclear euchromatin (G0:0005719) & jun \\
\hline & euchromatin (G0:0000791) & jun \\
\hline & nuclear chromosome (G0:0000228) & jun \\
\hline & chromosome (G0:0005694) & jun \\
\hline \multirow[t]{8}{*}{$\begin{array}{l}\text { Molecular } \\
\text { Function }\end{array}$} & $\begin{array}{l}\text { transcriptional repressor activity, RNA polymerase II activating transcription } \\
\text { factor binding (GO:0098811) }\end{array}$ & jun; \\
\hline & activating transcription factor binding (G0:0033613) & jun; \\
\hline & $\begin{array}{l}\text { transcriptional activator activity, RNA polymerase II transcription factor } \\
\text { binding (GO:0001190) }\end{array}$ & $\begin{array}{l}\text { jun; } \\
\text { Imo2 }\end{array}$ \\
\hline & cAMP response element binding (G0:0035497) & jun \\
\hline & RNA polymerase II activating transcription factor binding (G0:0001102) & $\begin{array}{l}\text { jun; } \\
\text { Imo2 }\end{array}$ \\
\hline & glycine binding (GO:0016594) & alas2 \\
\hline & R-SMAD binding (GO:0070412) & jun \\
\hline & $\begin{array}{l}\text { RNA polymerase II distal enhancer sequence-specific DNA binding } \\
\text { (GO:0000980) }\end{array}$ & jun \\
\hline
\end{tabular}




\section{Discussion}

Next-generation sequencing has been widely applied for global analysis in several studies involving miRNAs and skeletal muscle. Nachtigall et al. employed next-generation sequencing in Nile Tilapia (Oreochromis niloticus), in order to obtain information regarding evolutionary pathways with emphasis on muscle miRNAs [12]. The authors identified that there are large syntenic blocks in the genome, possibly being linked to a common function of such miRNAs, and as well specific role of miR-499 expression [15].

Previous studies in our group have also analyzed the function of some miRNAs in the skeletal muscle of fish involving fasting and re-feeding treatments and muscle development [16], [17]. The authors observed that some miRNAs (miR-1, miR-133, miR-155, miR-206, and miR-499) presented a possible role in the regulation of factors related to muscle cell proliferation and differentiation and with muscle performance and metabolism, modulating the rate of protein synthesis and degradation.

In the present study, we identified a small number of differentially expressed miRNAs considering the comparison between the genotypes pairs. The comparison between tambacu and P. mesopotamicus (TC vs. PC) showed $15 \mathrm{DE}$ miRNAs, 8 upregulated and 7 downregulated. Considering the comparison between tambacu and C.macropomum (TC vs. TQ), we observed 24 DE miRNAs, 14 upregulated and 10 downregulated, and considering the comparison between $P$. mesopotamicus and $C$. macropomum (PC vs. TQ), we observed 24 DE miRNAs, 15 upregulated and 9 downregulated. Interestingly, the majority of the miRNAs were specific for each genotype pair. No miRNA was shared between the 3 genotype pairs, both up and downregulated. Between the upregulated DE miRNAs, 9 overlapped between PC vs. TQ and TC vs. TQ and 2 overlap between TC vs. PC and TC vs. TQ. Considering the downregulated DE miRNAs, 3 overlap between PC vs. TQ and TC vs. TQ, and only one overlaps between TC vs. PC and TC vs. TQ. No miRNA was differentially expressed between all of the three comparison pairs at the same time. Some miRNAs show characteristics that make it possible to track their inheritance pattern by looking into the different groups. For example, some miRNAs appear to be exclusive for the comparison between TC vs. TQ (miR-187, miR-18a, miR-190a, miR-206-3p, miR-221-3p, miR-27b-3p, miR-29a, miR-363-3p, and miR727-5p), some of which were exclusive in the comparison between PC vs. TQ (miR-146b, miR-212-5p, miR-2188-3p, miR-26a-2-3p, and miR-489). Interestingly, some miRNAs presented a characteristic of being inherited specifically of one genotype, and also, there were some miRNAs that appeared to be up- or down-regulated only in the hybrid (miR-10a-5p, miR-144-3p, and miR-199-3-3p). These miRNAs presented (up or downregulated) behavior, exclusively in the hybrid, not differentially expressed between the parental genotypes.

Ontology analysis by Enrichr, considering the Danio rerio validated interaction data, showed that the experimentally validated target genes of the DE miRNAs were grouped into several Biological Processes, Cellular Components, and Molecular Functions categories. The most enriched processes were oxygen homeostasis, nuclear euchromatin and transcriptional repressor activity, and RNA polymerase II 
activating transcription factor binding, respectively. The more enriched genes in the signaling pathway involved in these processes were alas2, jun, and Imo2.

miR-206-3p, identified in the present study, was differentially expressed in the comparison pair TC vs. TQ, targets jun and vegfa genes. Jun is a member of the Activator Protein 1 (AP-1) transcription factor family that regulates cell proliferation and differentiation, apoptosis, cellular migration, inflammation, and cell-cell interaction [18]. On the other hand, vegfa is involved in vascular development and new blood vessel formation [19], [20] and stimulates endothelial cell migration by activating AP-1 transcription factor jun [21]. Skeletal muscle is the most abundant source of VEGFA [22], [23], and skeletal-musclespecific VEGFA knockout changed angiogenesis in muscle fibers [24].

As jun and vegfa genes are targets of miR-206-3p and, as this miRNA was observed to be differentially expressed in the comparison pair TC vs. TQ, this is an indication that this miRNA can represent a remarkable characteristic for the hybrid, since these genes are involved in the maintenance of blood irrigation that, in turn, controls the oxygen rates in the tissues.

The miRNA involved in the control of the alas2 and Imo2 genes was miR-144-3p. This miRNA appeared upregulated in the comparison pairs TC vs. PC and TC vs. TQ and did not appear in the comparison between the parental genotypes PC vs. TQ.

ALAS2 is one of the isozymes of ALAS (5-aminolevulinate synthase) involved in vertebrate heme biosynthesis. This gene is expressed preferentially in erythroid cell-specific mitochondrial enzymes [25], [26] and catalyzes the biosynthesis of bulk heme for hemoglobin production [27]-[30]. ALAS2 is also regulated by hypoxia-inducible factor HIF. Khenchaduri and colaborators observed the overexpression of ALAS2 in cardiac myoblasts submitted to chronic hypoxia, with a corresponding increase in cellular heme levels. They concluded that, similar to erythroid cells, ALAS2 is positively regulated by hypoxia in cardiac myoblasts with an increase in heme levels [31].

According to Zhang et al., the upregulation of alas 2 during hypoxia is directly mediated by a transcription factor, hypoxia-inducible factor 1 (Hif1) [32], and an increased Hif-1 that occurs under low oxygen concentration conditions also promotes the activation of genes involved in hypoxia, such as vegf [33].

Moreover, it has been shown that the transcription factor LIM domain only 2 (Lmo2) is required for angiogenesis and is important for angiogenic remodeling of the existing capillary network into mature blood vessels [34]-[36]. Besides, Lmo2 forms common transcription complexes with GATA-binding protein 2 (GATA2) and cooperatively regulate VEGF-induced angiogenesis [37].

Therefore, it is possible that the interaction between miR-144-3p and alas 2 and Imo2 genes can regulate important pathways related to muscle vascularization, oxygen homeostasis, and oxidative metabolism. We believe that this is an important characteristic that probably could provide, for the hybrid tambacu, more resistance to environments with lower oxygen content, once this miRNA was upregulated only in the hybrid, in the comparison pairs TC vs. PC and TC vs. TQ. 
In our study, miR-144-3p was identified as DE in TC vs. PC and TC vs. TQ, and the miR-206-3p described as DE only in the comparison pair TC vs. TQ demonstrated that these miRNAs may represent an important species-specific feature. The hybrid tambacu is a very popular fish in Brazil, especially in the outh and southeast regions and combines important characteristics for commercial fish farms, such as resistance to low temperatures during the tropical winter, similar to pacu, and faster growth, similar to tambaqui [1], [3]. Also, juvenile tambacu exhibits a total capacity for compensatory growth when submitted to short periods of food deprivation, followed by refeeding, a fact that could contribute to reducing production costs [38].

Although there are few studies showing the real potential of the hybrid for aquaculture production [38], [39], some of them have been done to better understand the genetics and molecular characteristics of this genotype. Recently, Gomes et al. used Next Generation Sequencing technologies for the analysis of muscle and skin transcriptome of $C$. macropomum and the hybrid tambacu, observed differences in the specificity and gene expression levels in both the muscle and skin of the hybrid tambacu and the parental tambaqui [40]. The authors demonstrated that in the muscle of tambaqui, up-regulated genes involved in muscle contraction, such as the myosin family, actin and myomesin, and catalytic protein genes. The hybrid tambacu muscle presented upregulated genes controlling oxidative stress, amino acid metabolism, the ubiquitin family, defense, and heat shock protein (HSP) family genes, involved in stress.

In our study, we can emphasize that the interaction of jun and vegfa genes with miR-206-3p (TC vs. TQ) and Imo2 and alas 2 genes with miR-144-3p (TC vs. PC and TC vs. TQ) could provide an evolutionary advantage to the hybrid tambacu once these genes are controlling pathways related to blood vessel modulation, oxygen homeostasis, and oxidative metabolism. Taking this into account, the differential regulation of these miRNAs may represent an explanation of why the hybrid has a better adaptation from exposure to hypoxic environments [34], [41]. Besides, it could indicate an important function of miRNAs miR-206-3p and miR-144-3p in the control of the robustness and faster growth of the hybrid tambacu [42].

In addition, the most significant molecular function enriched by the targets of the up-regulated miRNAs in the tambacu muscle was transcriptional repressor activity. The inhibition of this function reinforces our hypothesis that these miRNAs could be related to the robustness and faster growth of the hybrid, since their activity may promote enhanced transcriptional activity, which combined with translation, is an essential process for muscle hypertrophy and growth [43].

\section{Conclusions}

Using comparative global miRNA analysis in the hybrid tambacu and their parental genotypes Piaractus mesopotamicus and Colossoma macropomum, we demonstrated, for the first time, miRNAs differentially expressed in the skeletal muscle of the genotype comparison pairs (TC vs. PC, TC vs. TQ, and PC vs. TQ). Furthermore, we showed that the hybrid expressed miRNAs that control important molecular muscle markers with potential applications in aquaculture management systems. Our study also provides insight 
for further investigations involving the validation of these miRNAs and their signaling pathway components in muscle phenotype in these genotypes.

\section{Methods}

\section{Experimental Design, Sample Collection, Total RNA Extraction, and Small RNAs Sequencing}

Pure early juvenile fish Colossoma macropomum, Piaractus mesopotamicus, and hybrid tambacu were obtained at the Aquaculture Center of UNESP - CAUNESP, and were maintained in aerated tanks in the Agribusiness Technology Agency (APTA), Presidente Prudente, Sao Paulo, until fish attained $10 \mathrm{~g}$. Fish were kept in a natural photoperiod ( $12 \mathrm{~h}$ light/dark), and water temperature around $28^{\circ} \mathrm{C}$. Dissolved oxygen and $\mathrm{pH}$ were monitored daily, and ammonia, nitrite, and nitrate were monitored weekly. Fish were fed with Guabi-Pirá 28 fish feed. Excess benzocaine (concentration over $250 \mathrm{mg} / \mathrm{L}$ ) was applied to euthanize the animals. Afterward, body weight and total length were determined, and fast muscle samples ( $n=10$ for each genotype) were collected from the epaxial region near the dorsal fin. Muscle samples were then frozen in liquid nitrogen for molecular analysis.

Total RNA was isolated using TRIZOL (Invitrogen), following the manufacturer's specifications. The total extracted RNA was treated with DNase (Life Technologies, USA), following the manufacturer's specifications. All extracted RNA samples were analyzed by NanoVue Spectrophotometer (GE Healthcare) and Bioanalyzer (Agilent Technologies) for checking the total concentration and the RNA Integrity Number (RIN). The RNA extraction resulted in high quality RNA, where the lowest RIN obtained was 8.7, which is 0.7 points higher than the minimum limit used for next-generation sequencing. In such a way, 5 samples for every genotype were selected based on a RIN $\geq 8$ for sequencing. The samples were shipped to LC Sciences (Houston, TX, USA) in dry ice, and the entire process of library preparation and sequencing by Illumina platform was carried out. Libraries preparation were carried out using the TruSeq Small RNA Library Preparation Kit, and samples were run in 50 cycle single-end Illumina HiSeq 2500 Fast Mode. After sequencing, the data were downloaded through a link provided by the company.

\section{MiRNA analysis}

The processing pipeline consisted initially of analyzing the data regarding the read quality (Phred Score). For this purpose, the software FastQC (http://www.bioinformatics.babraham.ac.uk/projects/fastqc) version 0.10 .1 was used. After obtaining the sequencing quality information, adapters were removed using the software Fastx-toolkit (http://hannonlab.cshl.edu/fastx_toolkit/index.html). Reads not containing adapters were kept in libraries. Reads that, after removing the adapters, presented less than 17 nucleotides in length were permanently excluded. A filter based on read-quality parameters using the Fastx-toolkit software was also carried out. In such way, $90 \%$ of the read content has a phred score of at least 30 . Reads not satisfying these quality prerequisites were also permanently excluded. The libraries 
were then filtered by max length using a custom Perl script, where reads longer than 26 nucleotides were discarded. The remaining reads were used to obtain an expression profile of known miRNAs, based on known miRNAs lists of Danio rerio, using data from mature and precursor miRNAs obtained from the MirBase database version 22 (http://www.mirbase.org). For return more alignment than other species, we selected Danio rerio as a reference for this study, which is considered a good biological model.

For the differential expression analysis of miRNAs, mature sequences were aligned against the precursor sequences using GMAP [44] in order to create an annotation file in GFF format, making it possible to locate the mature sequences in the precursor sequences and differentiate alignments between the $5^{\prime}$ and 3' miRNAs.

After building the annotation file, the filtered reads were aligned against the precursor references using Hisat2 [45]. The software featureCounts [46] was applied over the alignments in order to collect the readcount for each miRNA aligned against the precursor reference and matching the GFF annotation file. The counts were then analyzed to detect differentially expressed (DE) miRNAs using the Bioconductor/R package DESeq2 [47].

The three different genotypes received specific names regarding its popular names: PC - Piaractus mesopotamicus (pַacu), TQ - Colossoma macropomum (țambaqui), and TC (hybrid tambaçu).

A priori, we performed contrasts between the hybrid and each parental species, always having the hybrid as a base of comparison (TC vs. PC and TC vs. TQ). A posteriori, for further understanding of the traits inherited by each parental, in terms of miRNA expression profiles, we also have run a contrast analysis between the two parental genotypes. Such a second contrast has pacu as a base of comparison (PC vs. TQ). In this way, three contrasts were obtained: TC vs. PC, TC vs. TQ, and PC vs. TQ (see Additional files 3-6).

In order to consider DE characteristics, we established that miRNAs presenting log2FoldChange (log2FC) $\leq-0.75$ and $\geq 0.75$, followed by adjusted $p$-value (padj) $\leq 0.05$, were considered differentially expressed as downregulated and upregulated, respectively.

The list of DE miRNAs detected by DESeq2 analysis was applied to the drawing of Venn Diagrams in order to visualize the DE miRNA sharing characteristics of each genotype by using Venn 2.1.0 (http://bioinfogp.cnb.csic.es/tools/venny/). Venn diagrams were created in two ways, i.e., up-regulated miRNAs and down-regulated miRNAs.

The R package gplots was applied with the DE miRNAs list for obtain heatmaps, which gives us a graphical idea of the expression variations between all the contrasts.

Target genes were detected using MirTarBase [13] database and the results were applied to ontology analysis by using Enrichr [14] in order to retrieve information regarding pathways in which the target genes are involved. The MirTarBase search comprises only experimentally validated interactions classified as "strong evidence". 
Interaction networks were assembled using Cytoscape 3.7.1 [48] based on MirTarBase recovered data for a better observation of the interaction between DE miRNAs and their validated targets. Two sets of networks have been assembled: (i) a network containing validated interactions for Homo sapiens, Mus musculus, Rattus norvegicus, and Danio rerio, and (ii) a network containing only Danio rerio validated interactions.

\section{Abbreviations}

PC - Pacu

TQ - Tambaqui

TC - Hybrid tambacu

DE miRNAs - Differentially expressed miRNAs

PCA - Principal Component Analysis

vcana - Versican a

vegfa - Vascular Endotelial Growth Factor A

jun - AP-1 Transcription factor Subunit

cyb561d2 - Cytochrome B561 Family Member D2

Imo2 - LIM Domain Only 2

klfd-Kruppel-like factor d

gata2a-GATA-binding protein 2

meis 1 - Meis Homeobox 1

klf3 - Kruppel Like Factor 3

alas2- 5-aminolevulinate synthase 2

hif1 - Hypoxia-Inducible Factor 1

COBEA - Brazilian College of Animal Experimentation

APTA - Agribusiness Technology Agency

RIN - RNA Integrity Number 


\section{Declarations}

\section{Availability of data and materials}

All raw and processed data have been deposited on the GEO dataset, under access number GSE147532.

The reviewers can access the data using the following token: kfcpcscsdhcffkx.

\section{Ethics approval and consent to participate}

This study was approved by the Ethics Committee on Animal Use - CEEA/UNESP, Institute of Biosciences, Botucatu, SP (Protocol. 1002 - CEUA) and was performed in accordance with the Ethical

Principles in Animal Research adopted by the Brazilian College of Animal Experimentation (COBEA). The experiments were also conducted following the ARRIVE guidelines [49].

\section{Consent for publication}

All authors read and are consent to publish the data.

\section{Funding}

BEAF and MDPS were supported by FAPESP Proc. 2015/19985-2, Proc. 2016/05009-4 and Proc. 2017/15708-0. MDPS also was supported by CNPq, Proc. 302656/2015-4 as research scholarship.

\section{Competing interests}

The authors declare that they have no competing interests.

\section{Authors" contributions}

Conceived and designed the experiments: BEAF, RFC and MDPS.

Performed the experiments: BEAF, ESP, JSV, TGP and BTTZ.

Responsible for the bioinformatics analysis: BEAF, SP, MAD.

Data organization: BEAF, ESP, BTTZ, SP, MAD, RFC and MDPS.

Responsible for fish husbandry: BEAF, EAM and MDPS.

Wrote and reviewed the manuscript: BEAF, ESP, BTTZ, JSV, TGP, RFC, SP, MAD and MDPS. 


\section{Acknowledgements}

We are thankful to UNESP and CIBIO for providing the structure needed. We are thankful to FAPESP and CNPq for providing the finalcial support. We are thankful to Rondinelle Simões Salomão for carrying out the morphological classification of the three genotypes analyzed.

\section{References}

1. C. Araújo-Lima and M. Goulding, Os frutos do tambaqui: ecologia, conservação e cultivo na Amazônia. 1998.

2. D. M. Bartley, K. Rana, and A. J. Immink, "The use of inter-specific hybrids in aquaculture and fisheries," Rev. Fish Biol. Fish., vol. 10, no. 3, pp. 325-337, 2000.

3. M. Tavares-dias, F. R. De Moraes, E. Makoto, and P. C. B. Rezende, "Changes in blood parameters of hybrid tambacu fish parasitized by Dolops carvalhoi (Crustacea , Branchiura ), a fish louse," Vet. Arh., vol. 77, no. 4, pp. 355-363, 2007.

4. A. L. Alves, E. S. Varela, G. V. Moro, and L. N. Kirschnik, "Riscos genéticos da produção de híbridos de peixes nativos," Embrapa Pesca e Aquicultura, p. 60, 2014.

5. A. Listrat et al., "How muscle structure and composition determine meat quality," Prod. Anim., vol. 28, no. 2, pp. 125-136, 2015.

6. J. Pal, B. N. Shukla, A. K. Maurya, and H. O. Verma, "A review on role of fish in human nutrition with special emphasis to essential fatty acid," Int. J. Fish. Acquat. Stud., vol. 6, no. 2, pp. 427-430, 2018.

7. K. V. Morris and J. S. Mattick, "The rise of regulatory RNA," Nat. Rev. Genet., vol. 15, no. 6, pp. 423437, 2014.

8. J. Rinn and M. Guttman, "RNA and dynamic nuclear organization," Science (80-. )., vol. 345, no. 6202, pp. 1240-1241, Sep. 2014.

9. D. P. Bartel, “MicroRNAs," Cell, vol. 116, no. 2, pp. 281-297, Jan. 2004.

10. D. P. Bartel, “MicroRNAs: Target Recognition and Regulatory Functions," Cell, vol. 136, no. 2, pp. 215233, Jan. 2009.

11. J. J. McCarthy, "The MyomiR Network in Skeletal Muscle Plasticity," Exerc. Sport Sci. Rev., vol. 39, no. 3, pp. 150-154, Jul. 2011.

12. P. G. Nachtigall, M. C. Dias, and D. Pinhal, "Evolution and genomic organization of muscle microRNAs in fish genomes," BMC Evol. Biol., vol. 14, no. 1, pp. 1-12, 2014.

13. C.-H. Chou et al., "miRTarBase update 2018: a resource for experimentally validated microRNA-target interactions," Nucleic Acids Res., vol. 46, no. D1, pp. D296-D302, Jan. 2018.

14. E. Y. Chen et al., "Enrichr: interactive and collaborative HTML5 gene list enrichment analysis tool," BMC Bioinformatics, vol. 14, no. 1, p. 128, Apr. 2013.

15. P. G. Nachtigall, M. C. Dias, R. F. Carvalho, C. Martins, and D. Pinhal, "MicroRNA-499 Expression Distinctively Correlates to Target Genes sox6 and rod1 Profiles to Resolve the Skeletal Muscle 
Phenotype in Nile Tilapia," PLoS One, vol. 10, no. 3, p. e0119804, Mar. 2015.

16. B. O. D. S. Duran et al., "Differential microRNA Expression in Fast- and Slow-Twitch Skeletal Muscle of Piaractus mesopotamicus during Growth," PLoS One, vol. 10, no. 11, p. e0141967, Nov. 2015.

17. T. G. de Paula et al., "Food restriction increase the expression of mTORC1 complex genes in the skeletal muscle of juvenile pacu (Piaractus mesopotamicus)," PLoS One, vol. 12, no. 5, p. e0177679, May 2017.

18. E. Shaulian, "AP-1 - The Jun proteins: Oncogenes or tumor suppressors in disguise?," Cell. Signal., vol. 22, no. 6, pp. 894-899, 2010.

19. H. F. Dvorak, M. Detmar, K. P. Claffey, J. A. Nagy, L. Van De Water, and D. R. Senger, "Vascular permeability factor/ vascular endothelial growth factor: An important mediator of angiogenesis in malignancy and inflammation," Int. Arch. Allergy Immunol., vol. 107, no. 1-3, pp. 233-235, 1995.

20. S. Choorapoikayil, B. Weijts, R. Kers, A. de Bruin, and J. den Hertog, "Loss of Pten promotes angiogenesis and enhanced vegfaa expression in zebrafish," Dis. Model. Mech., vol. 6, no. 5, pp. 1159-1166, 2013.

21. J. Jia, T. Ye, P. Cui, Q. Hua, H. Zeng, and D. Zhao, "AP-1 transcription factor mediates VEGF-induced endothelial cell migration and proliferation," Microvasc. Res., vol. 105, pp. 103-108, 2016.

22. E. A. Logsdon, S. D. Finley, A. S. Popel, and F. MacGabhann, "A systems biology view of blood vessel growth and remodelling," J. Cell. Mol. Med., vol. 18, no. 8, pp. 1491-1508, 2014.

23. P. Yen, S. D. Finley, M. O. Engel-Stefanini, and A. S. Popel, “A two-compartment model of VEGF distribution in the mouse," PLoS One, vol. 6, no. 11, 2011.

24. K. Tang, E. C. Breen, H. P. Gerber, N. M. A. Ferrara, and P. D. Wagner, "Capillary regression in vascular endothelial growth factor-deficient skeletal muscle," Physiol. Genomics, vol. 18, pp. 63-69, 2004.

25. M. Yamamoto, N. S. Yew, M. Federspiel, J. B. Dodgson, N. Hayashi, and J. D. Engel, "Isolation of recombinant cDNAs encoding chicken erythroid delta-aminolevulinate synthase.," Proc. Natl. Acad. Sci., vol. 82, no. 11, pp. 3702-3706, Jun. 1985.

26. R. D. Riddle, M. Yamamoto, and J. D. Engel, “Expression of delta-aminolevulinate synthase in avian cells: separate genes encode erythroid-specific and nonspecific isozymes., Proc. Natl. Acad. Sci., vol. 86, no. 3, pp. 792-796, Feb. 1989.

27. O. Nakajima et al., "Heme deficiency in erythroid lineage causes differentiation arrest and cytoplasmic iron overload," EMBO J., vol. 18, no. 22, pp. 6282-6289, Nov. 1999.

28. O. Nakajima et al., "Transgenic rescue of erythroid 5-aminolevulinate synthase-deficient mice results in the formation of ring sideroblasts and siderocytes," Genes to Cells, vol. 11, no. 6, pp. 685-700, Jun. 2006.

29. M. Yamamoto and O. Nakajima, "Animal models for X-linked sideroblastic anemia.," Int. J. Hematol., vol. 72, no. 2, pp. 157-64, Aug. 2000.

30. G. A. Hunter and G. C. Ferreira, "5-Aminolevulinate synthase: Catalysis of the first step of heme biosynthesis," Cellular and Molecular Biology, vol. 55, no. 1. pp. 102-110, 2009. 
31. A. Khechaduri, M. Bayeva, H. C. Chang, and H. Ardehali, "Heme levels are increased in human failing hearts," J. Am. Coll. Cardiol., vol. 61, no. 18, pp. 1884-1893, May 2013.

32. F.-L. Zhang et al., "Hypoxic Induction of Human Erythroid-Specific $\delta$-Aminolevulinate Synthase Mediated by Hypoxia-Inducible Factor 1," Biochemistry, vol. 50, no. 7, pp. 1194-1202, Feb. 2011.

33. K. Salnikow et al., "The Regulation of Hypoxic Genes by Calcium Involves c-Jun/AP-1, Which Cooperates with Hypoxia-Inducible Factor 1 in Response to Hypoxia," Mol. Cell. Biol., vol. 22, no. 6, pp. 1734-1741, 2002.

34. Y. Yamada, R. Pannell, A. Forster, and T. H. Rabbitts, "The oncogenic LIM-only transcription factor Lmo2 regulates angiogenesis but not vasculogenesis in mice," Proc. Natl. Acad. Sci., vol. 97, no. 1, pp. 320-324, Jan. 2000.

35. D. Gratzinger et al., "The Transcription Factor LMO2 Is a Robust Marker of Vascular Endothelium and Vascular Neoplasms and Selected Other Entities," Am. J. Clin. Pathol., vol. 131, no. 2, pp. 264278, Feb. 2009.

36. S. Meng, G. Matrone, J. Lv, K. Chen, W. T. Wong, and J. P. Cooke, "LIM domain only 2 regulates endothelial proliferation, angiogenesis, and tissue regeneration," J. Am. Heart Assoc., vol. 5, no. 10, 2016.

37. S. Coma, M. Allard-Ratick, T. Akino, L. A. van Meeteren, A. Mammoto, and M. Klagsbrun, "GATA2 and Lmo2 control angiogenesis and lymphangiogenesis via direct transcriptional regulation of neuropilin-2," Angiogenesis, vol. 16, no. 4, pp. 939-952, Oct. 2013.

38. A. de L. Paz, Y. M. Pastrana, and L. V. Brandão, "Food deprivation does not affect growth performance of juvenile tambacu," Acta Amaz., vol. 48, no. 3, pp. 207-210, 2018.

39. R. Macedo Silva, M. Tavares-Dias, M. W. Reis Dias, M. K. Reis Dias, and R. das Graças Barbosa Marinho, "Parasitic fauna in hybrid tambacu from fish farms," Pesqui. Agropecu. Bras., vol. 48, no. 8, pp. 1049-1057, 2013.

40. F. Gomes et al., "Comparative analysis of the transcriptome of the Amazonian fish species Colossoma macropomum (tambaqui) and hybrid tambacu by next generation sequencing," PLoS One, vol. 14, no. 2, pp. 1-23, 2019.

41. A. J. Warren, W. H. Colledge, M. B. L. Carlton, M. J. Evans, A. J. H. Smith, and T. H. Rabbitts, "The Oncogenic Cysteine-rich LIM domain protein Rbtn2 is essential for erythroid development," Cell, vol. 78, no. 1, pp. 45-57, Jul. 1994.

42. J. S. C. Melo and J. A. Pereira, "Crescimento do Híbrido Tambacu (Fêmea de Colossoma macropomum X macho de Piaractus mesopotamicus) em criação intensiva," vol. 7, pp. 59-75, 1994.

43. F. Von Walden, "Ribosome biogenesis in skeletal muscle: Coordination of transcription and translation," Journal of Applied Physiology, vol. 127, no. 2. pp. 591-598, 2019.

44. T. D. Wu and C. K. Watanabe, "GMAP: a genomic mapping and alignment program for mRNA and EST sequences," Bioinformatics, vol. 21, no. 9, pp. 1859-1875, May 2005.

45. D. Kim, B. Langmead, and S. L. Salzberg, "HISAT: A fast spliced aligner with low memory requirements," Nat. Methods, vol. 12, no. 4, pp. 357-360, 2015. 
46. Y. Liao, G. K. Smyth, and W. Shi, "featureCounts: an efficient general purpose program for assigning sequence reads to genomic features," Bioinformatics, vol. 30, no. 7, pp. 923-930, Apr. 2014.

47. M. I. Love, W. Huber, and S. Anders, "Moderated estimation of fold change and dispersion for RNAseq data with DESeq2," Genome Biol., vol. 15, no. 12, p. 550, Dec. 2014.

48. P. Shannon, "Cytoscape: A Software Environment for Integrated Models of Biomolecular Interaction Networks," Genome Res., vol. 13, no. 11, pp. 2498-2504, Nov. 2003.

49. C. Kilkenny, W. J. Browne, I. C. Cuthill, M. Emerson, and D. G. Altman, "Improving bioscience research reporting: The arrive guidelines for reporting animal research," PLoS Biol., vol. 8, no. 6, pp. 1-2, 2010.

\section{Figures}

\section{Reads filtering by Group}

\section{0}

60000000

50000000

40000000

30000000

20000000

10000000

0

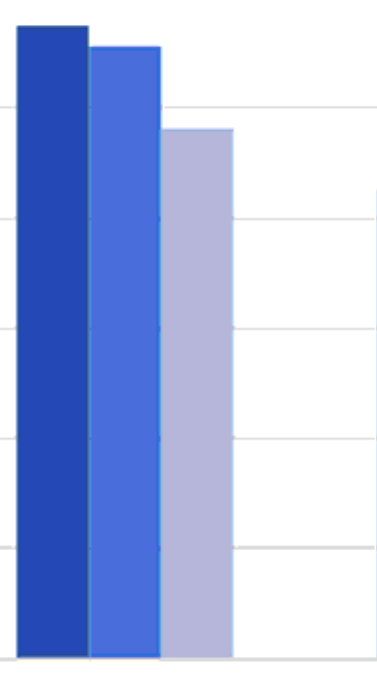

Raw

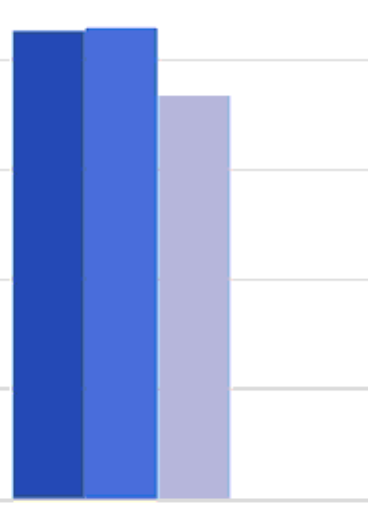

Clipped

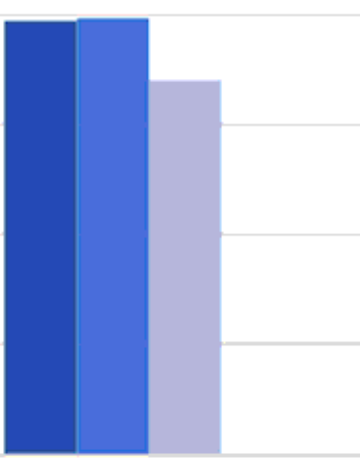

Filtered
- $\mathrm{PC}$ - TC TQ

\section{Figure 1}

Bar graphs showing the read numbers throughout the filtering process. Raw (raw data after downloading), Clipped (remaining reads after removing adaptors and reads shorter than 17nt), Filtered (remaining reads after filtering by read quality) and Sized (remaining reads after removing sequences longer than 26nt). 


\section{Principal Component Analysis}

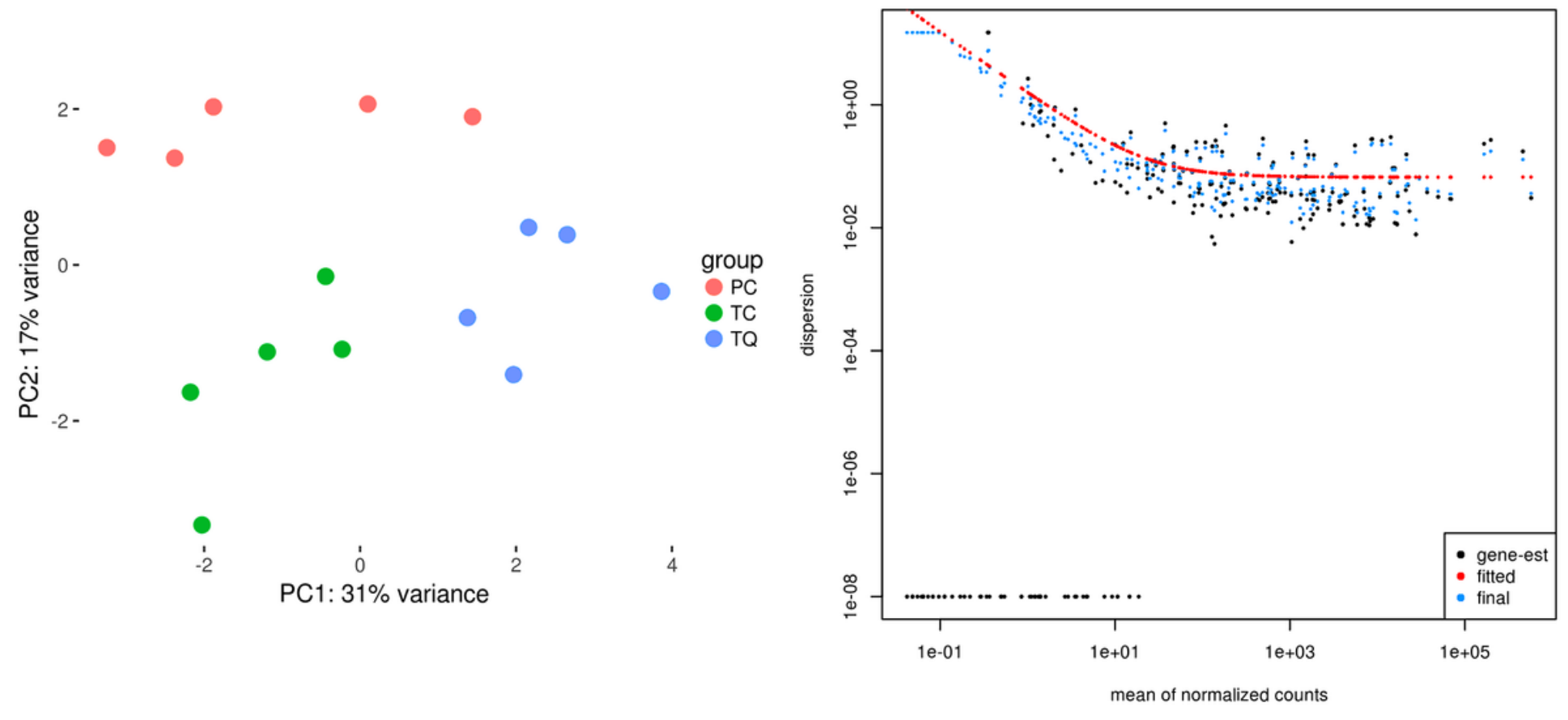

Figure 2

A: Principal Component Analysis obtained using the full log-transformed list of mean read-count miRNAs showing the different grouping samples. B: Dispersion Plot showing high dispersion values below a mean count of 10 . 


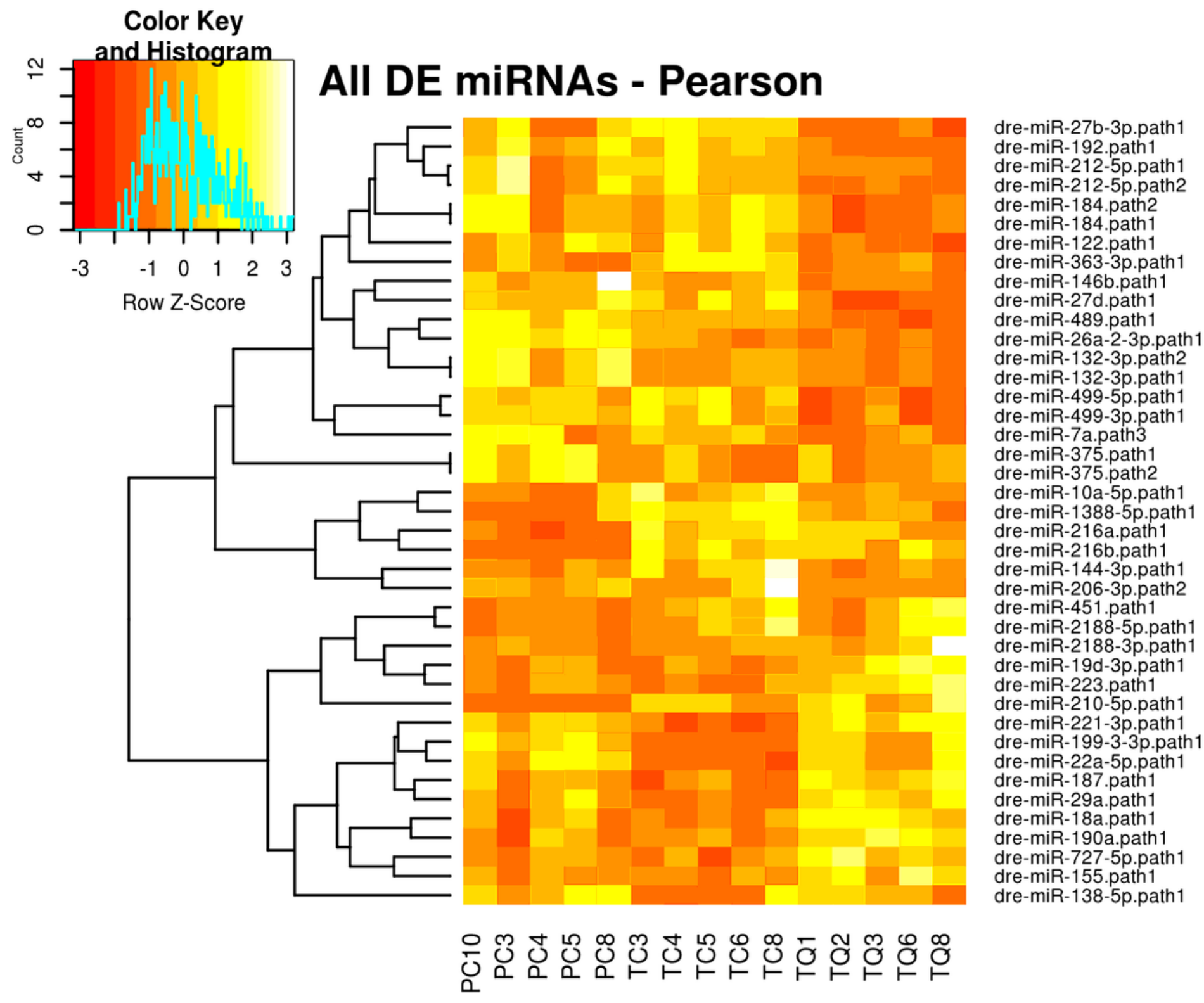

Figure 3

Row-clusterized heatmap showing differentially expressed miRNAs between all groups. Light colors, high expressed. Dark colors, low expressed. Rows were clusterized using Pearson correlation. 


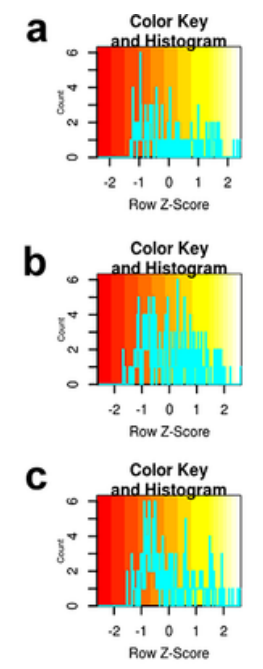

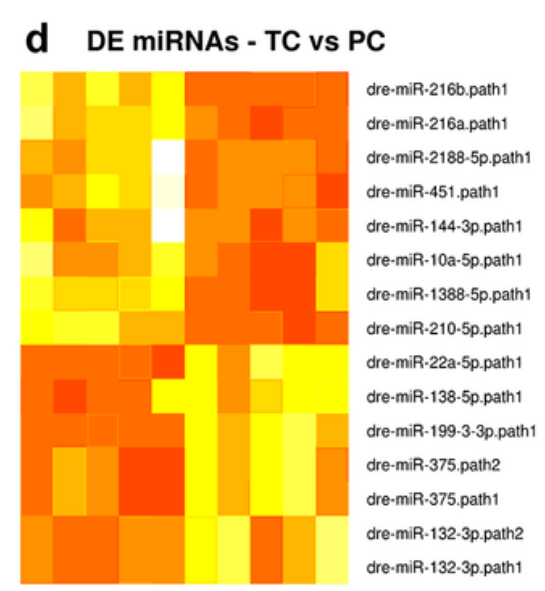

e DE miRNAs - TC vs TQ

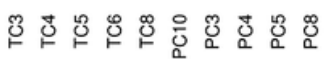

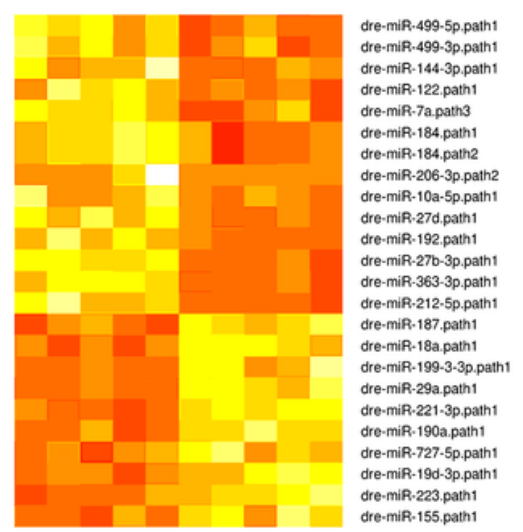

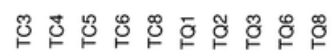

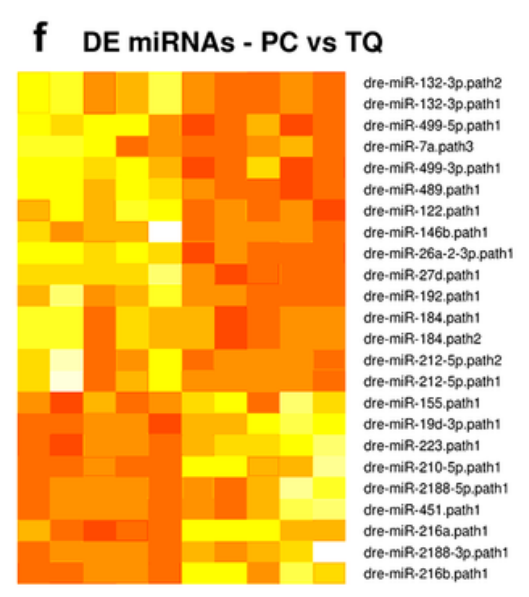

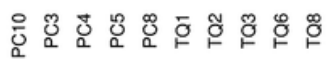

Figure 4

Non-clusterized heatmap showing differentially expressed miRNAs between groups. TC vs. PC (a and d), TC vs. TQ (b and e), and PC vs. TQ (c and f). Light colors, high expressed. Dark colors, low expressed. miRNAs have been ordered according to a decreasing Log2FC.

TC vs PC

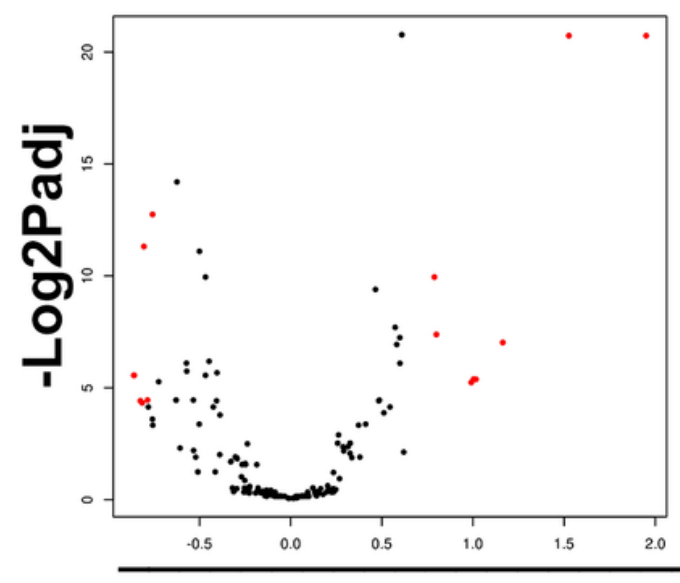

TC vs TQ

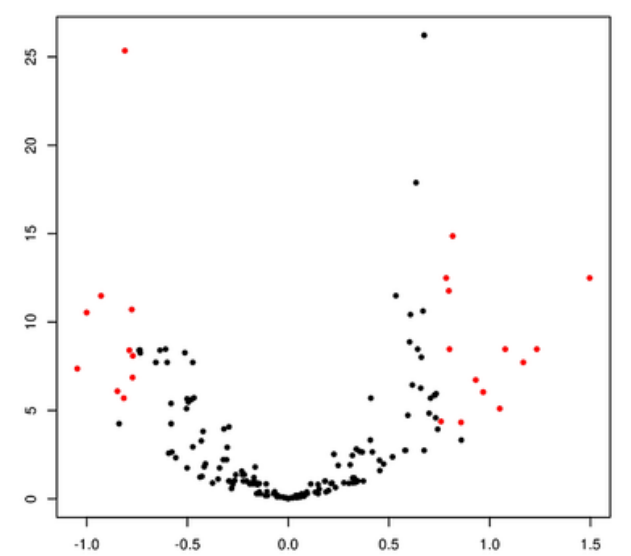

PC vs TQ

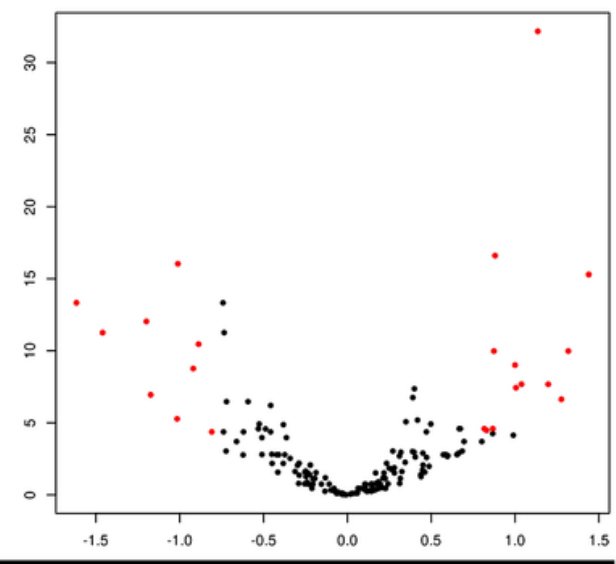

\section{Log2FC}

Figure 5

Volcano Plots showing differentially expressed miRNAs between the comparisons. Log2FC $\geq 0.75$ and padj $\leq$ 0.05. X axis, Log2FC. Y axis, -Log2padj. Differentially expressed miRNAs (DE miRNAs) are shown in red color. 
PC.TQ only

dre-miR-132-3p.path2

dre-miR-132-3p.path1

dre-miR-489.path1

dre-miR-146b.path1

dre-miR-26a-2-3p. path1

dre-miR-212-5p.path2
PC.TQ and TC.PC

zero

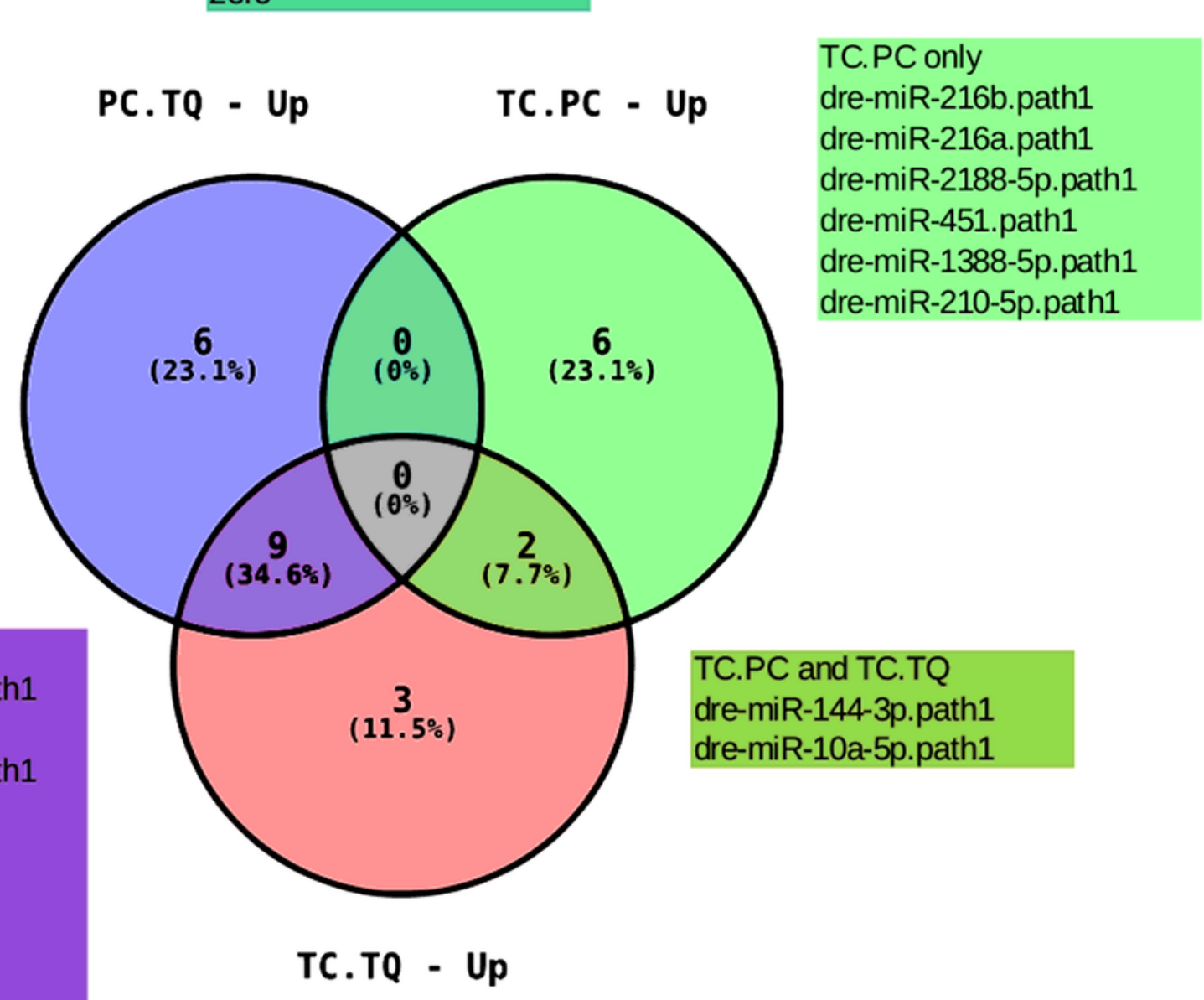

TC.TQ only

Dre-miR-206-3p.path2

dre-miR-27b-3p.path1

dre-miR-363-3p.path1

Figure 6

Venn Diagram showing the relationship between up-regulated miRNAs throughout the different groups.

The number marked in the overlapping areas shows the common DE miRNAs. 


PC.TQ only
dre-miR-210-5p.path1
dre-miR-2188-5p.path1
dre-miR-451.path1
dre-miR-216a.path1
dre-miR-2188-3p.path1
dre-miR-216b.path1

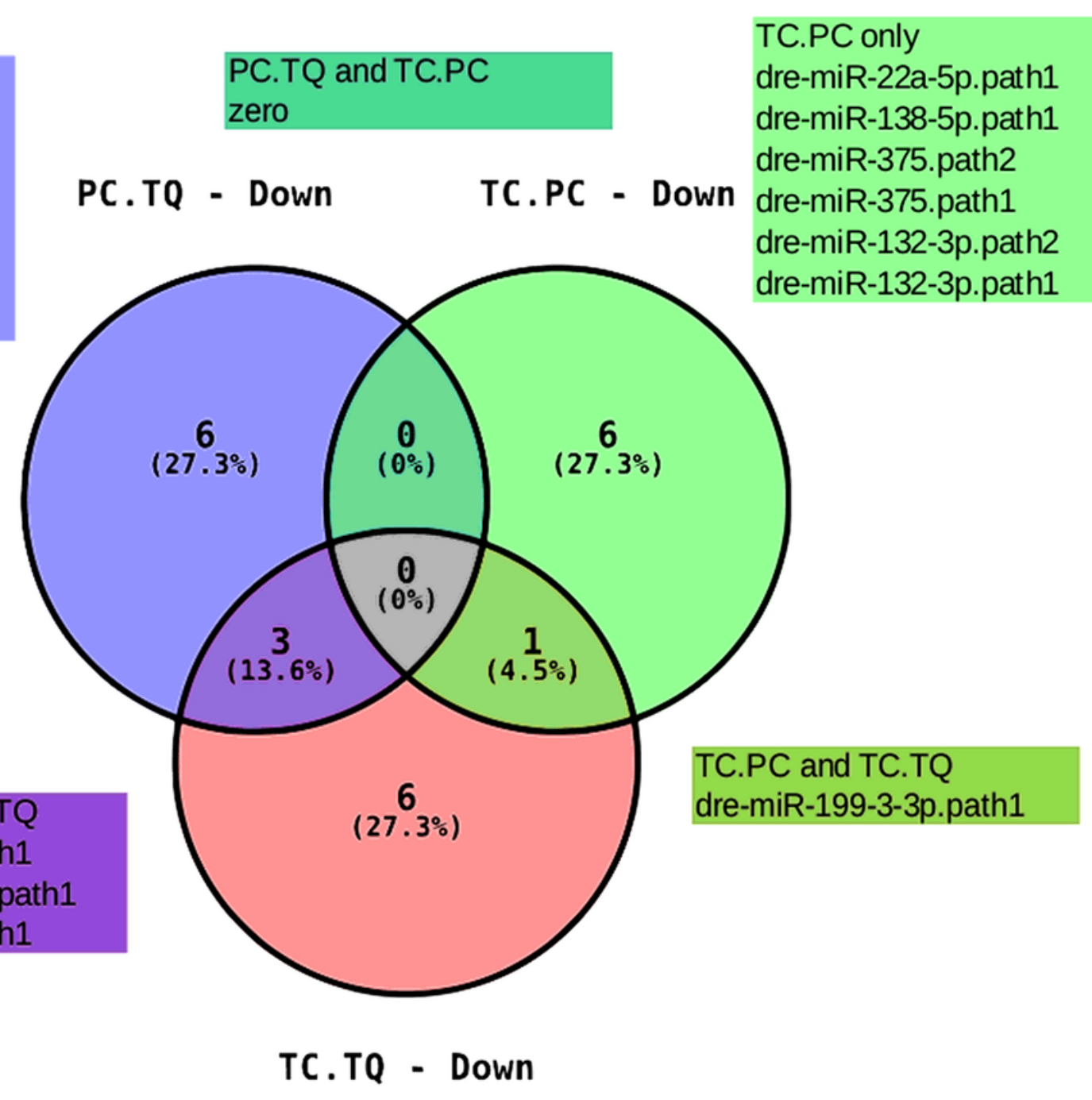

TC.TQ and PC.TQ dre-miR-155. path1 dre-miR-19d-3p.path1 dre-miR-223.path1

TC.TQ only
dre-miR-187.path1
dre-miR-18a.path1
dre-miR-29a.path1
dre-miR-221-3p.path1
dre-miR-190a.path1
dre-miR-727-5p.path1

Figure 7

Venn Diagram showing the relationship between down-regulated miRNAs throughout the different groups. The number marked in the overlapping areas shows the common DE miRNAs. 


\begin{tabular}{|c|c|c|c|c|}
\hline & miRNAs & TC vs $\mathrm{P}$ & TC vs TQ & $P C$ vs TQ \\
\hline 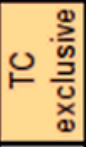 & $\begin{array}{l}\text { dre-miR-10a-5p. path1 } \\
\text { dre-miR-144-3p.path1 } \\
\text { dre-miR-199-3-3p.path1 }\end{array}$ & $\begin{array}{l}\Delta \\
\Delta \\
\nabla\end{array}$ & $\begin{array}{l}\Delta \\
\Delta \\
\nabla\end{array}$ & na \\
\hline 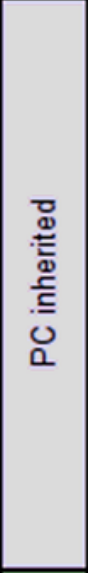 & $\begin{array}{l}\text { dre-miR-122.path1 } \\
\text { dre-miR-155. path1 } \\
\text { dre-miR-184.path1 } \\
\text { dre-miR-184.path2 } \\
\text { dre-miR-192.path1 } \\
\text { dre-miR-19d-3p.path1 } \\
\text { dre-miR-212-5p.path1 } \\
\text { dre-miR-223.path1 } \\
\text { dre-miR-27d.path1 } \\
\text { dre-miR-499-3p.path1 } \\
\text { dre-miR-499-5p.path1 } \\
\text { dre-miR-7a.path3 }\end{array}$ & na & $\begin{array}{l}\Delta \\
\boldsymbol{\nabla} \\
\Delta \\
\Delta \\
\Delta \\
\boldsymbol{\nabla} \\
\Delta \\
\boldsymbol{\nabla} \\
\Delta \\
\Delta \\
\Delta \\
\Delta \\
\Delta\end{array}$ & $\begin{array}{l}\boldsymbol{\Delta} \\
\boldsymbol{\nabla} \\
\boldsymbol{\Delta} \\
\boldsymbol{\Delta} \\
\boldsymbol{\Delta} \\
\boldsymbol{\nabla} \\
\Delta \\
\boldsymbol{\nabla} \\
\Delta \\
\Delta \\
\Delta \\
\Delta \\
\Delta \\
\end{array}$ \\
\hline 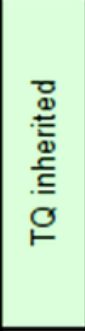 & $\begin{array}{l}\text { dre-miR-132-3p.path1 } \\
\text { dre-miR-132-3p.path2 } \\
\text { dre-miR-210-5p.path1 } \\
\text { dre-miR-216a.path1 } \\
\text { dre-miR-216b.path1 } \\
\text { dre-miR-2188-5p.path1 } \\
\text { dre-miR-451.path1 }\end{array}$ & $\begin{array}{l}\boldsymbol{\nabla} \\
\boldsymbol{\nabla} \\
\Delta \\
\Delta \\
\Delta \\
\Delta \\
\Delta \\
\Delta\end{array}$ & na & $\begin{array}{l}\boldsymbol{\Delta} \\
\boldsymbol{\Delta} \\
\boldsymbol{\nabla} \\
\boldsymbol{\nabla} \\
\boldsymbol{\nabla} \\
\boldsymbol{\nabla} \\
\boldsymbol{\nabla}\end{array}$ \\
\hline 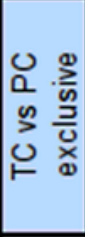 & $\begin{array}{l}\text { dre-miR-138-5p.path1 } \\
\text { dre-miR-1388-5p.path1 } \\
\text { dre-miR-22a-5p.path1 } \\
\text { dre-miR-375.path1 } \\
\text { dre-miR-375.path2 }\end{array}$ & $\begin{array}{l}\boldsymbol{\nabla} \\
\boldsymbol{\Delta} \\
\boldsymbol{\nabla} \\
\boldsymbol{\nabla} \\
\boldsymbol{\nabla}\end{array}$ & & \\
\hline 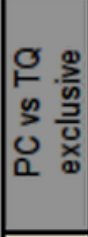 & $\begin{array}{l}\text { dre-miR-146b.path1 } \\
\text { dre-miR-212-5p.path2 } \\
\text { dre-miR-2188-3p.path1 } \\
\text { dre-miR-26a-2-3p.path1 } \\
\text { dre-miR-489.path1 }\end{array}$ & \multicolumn{2}{|c|}{ na } & $\begin{array}{l}\Delta \\
\Delta \\
\boldsymbol{\nabla} \\
\Delta \\
\Delta\end{array}$ \\
\hline 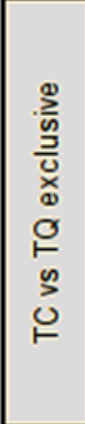 & $\begin{array}{l}\text { dre-miR-187. path1 } \\
\text { dre-miR-18a.path1 } \\
\text { dre-miR-190a. path1 } \\
\text { dre-miR-206-3p.path2 } \\
\text { dre-miR-221-3p.path1 } \\
\text { dre-miR-27b-3p.path1 } \\
\text { dre-miR-29a.path1 } \\
\text { dre-miR-363-3p.path1 } \\
\text { dre-miR-727-5p.path1 }\end{array}$ & na & $\begin{array}{l}\boldsymbol{\nabla} \\
\boldsymbol{\nabla} \\
\boldsymbol{\nabla} \\
\boldsymbol{\Delta} \\
\boldsymbol{\nabla} \\
\boldsymbol{\Delta} \\
\boldsymbol{\nabla} \\
\boldsymbol{\Delta} \\
\boldsymbol{\nabla}\end{array}$ & na \\
\hline
\end{tabular}

\section{Figure 8}

miRNA profiles summary between species. $\boldsymbol{\Delta}$ and $\boldsymbol{\nabla}$ : Up- and Down-regulated, respectively. Na: not applicable. MiRNAs were added to six different categories based on their differential expression characteristics. If a given miRNA appears up- or down-regulated only in the hybrid, it presents a hybridexclusive behavior. If a given miRNA is up- or down-regulated in the one parental and in the hybrid, it presents a behavior inherited from that parental. 


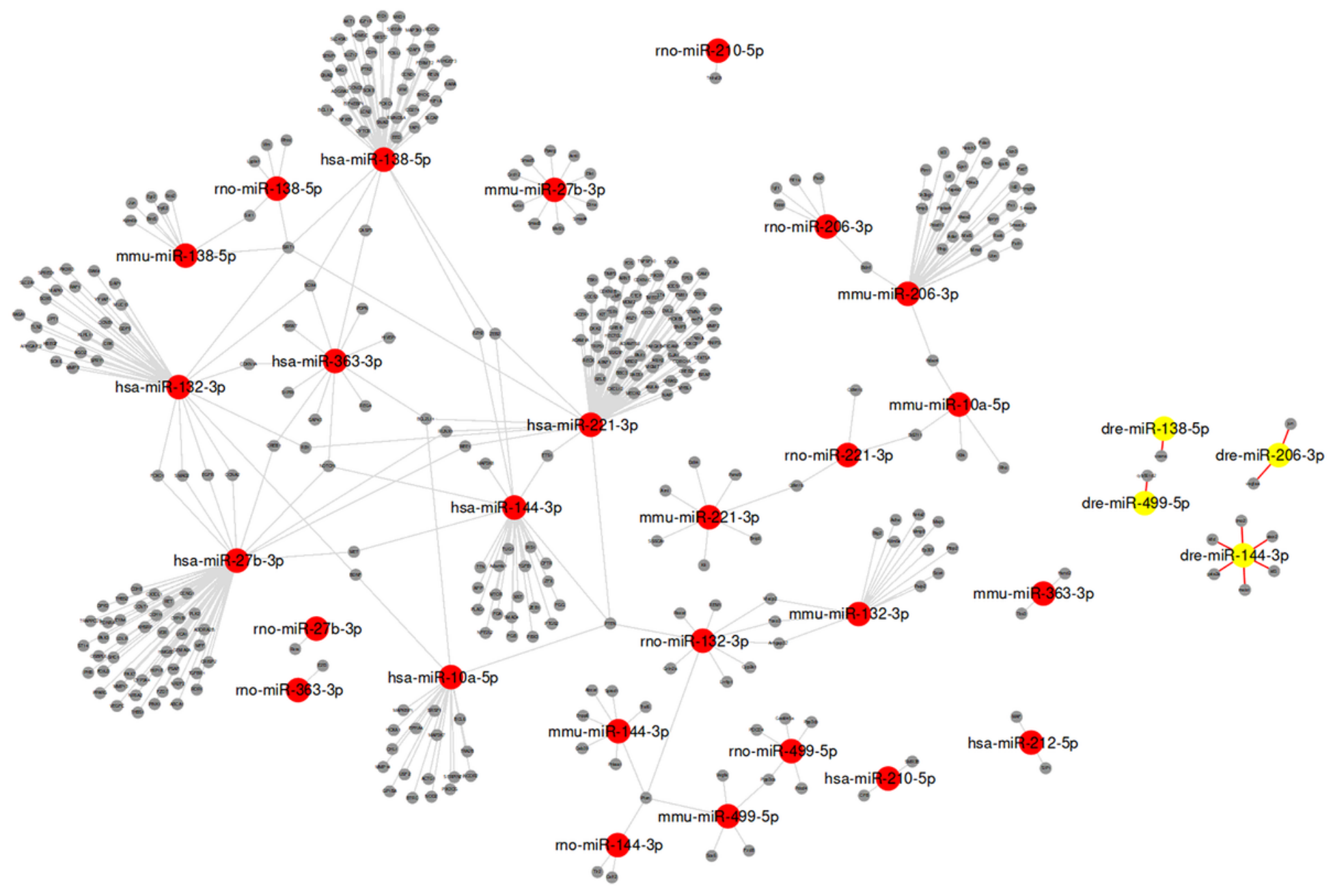

\section{Figure 9}

Experimentally validated interactions among DE miRNAs based on Danio rerio, Homo sapiens, Mus musculus and Rattus novergicus. Danio rerio interaction data is shown in yellow nodes. 


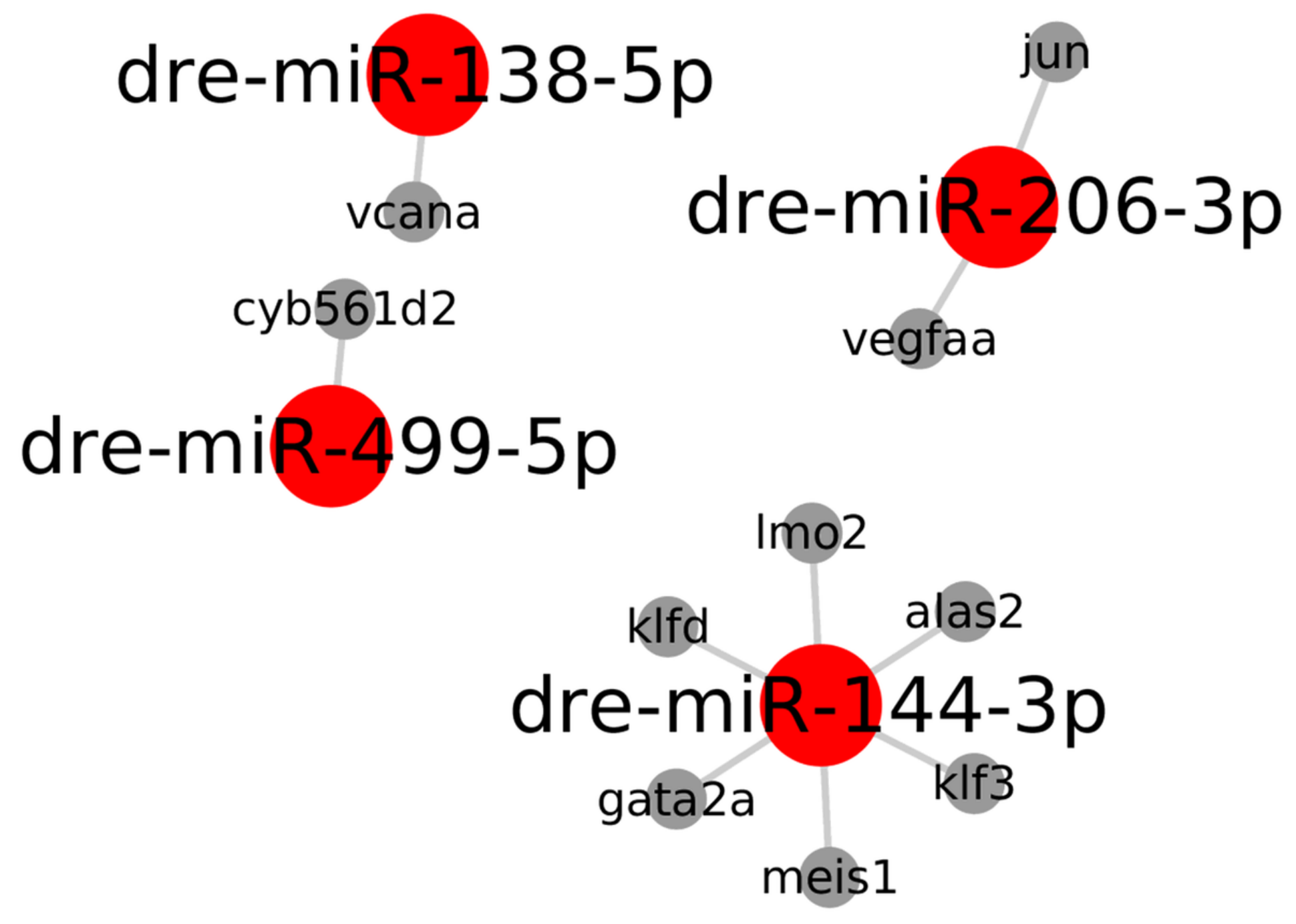

Figure 10

Experimentally validated interactions among DE miRNAs based on Danio rerio. vcana - Versican a; cyb561d2 - Cytochrome B561 Family Member D2; jun - AP-1 Transcription factor Subunit; vegfa Vascular Endotelial Growth Factor A; Imo2 - LIM Domain Only 2; klfd - Kruppel-like factor d; gata2a GATA-binding protein 2; meis 1 - Meis Homeobox 1; klf3 - Kruppel Like Factor 3; alas2- 5-aminolevulinate synthase 2; hif1 - Hypoxia-Inducible Factor 1. 


\section{gas homeostasis (GO:0033483)}

cellular response to thyroid hormone stimulus (GO:0097067)

negative regulation of transcription from RNA polymerase II promoter in response to stress (GO:0097201)

regulation of DNA-templated transcription, initiation (GO:2000142)

mRNA transcription from RNA polymerase II promoter (GO:0042789)

negative regulation of hemopoiesis (GO:1903707)

negative regulation by host of viral transcription (GO:0043922)

mRNA transcription (GO:0009299)

positive regulation of vascular smooth muscle cell proliferation (GO:1904707)

\section{Figure 11}

Biological processes involved with validated-target genes of the DE miRNAs. Upper bars represent more significant data. Lower bars represent less significant data.

nuclear euchromatin (GO:0005719)

euchromatin (GO:0000791)

nuclear chromosome (GO:0000228)

chromosome (GO:0005694)

RNA polymerase II transcription factor complex (GO:0090575)

nuclear chromatin (GO:0000790)

chromatin (GO:0000785)

mitochondrial inner membrane (GO:0005743)

mitochondrial matrix (GO:0005759)

nuclear chromosome part (GO:0044454)

\section{Figure 12}

Cellular components involved with validated-target genes of the DE miRNAs. Upper bars represent more significant data. Lower bars represent less significant data. 


\section{activating transcription factor binding (GO:0033613)}

transcriptional activator activity, RNA polymerase II transcription factor binding (GO:0001190)

\section{CAMP response element binding (GO:0035497)}

RNA polymerase II activating transcription factor binding (GO:0001102)

glycine binding (GO:0016594)

R-SMAD binding (60:0070412)

RNA polymerase II distal enhancer sequence-specific DNA binding (GO:0000980)

bHLH transcription factor binding (GO:0043425)

RNA polymerase II transcription factor binding (GO:0001085)

\section{Figure 13}

Molecular functions involved with validated-target genes of the DE miRNAs. Upper bars represent more significant data. Lower bars represent less significant data.

\section{Supplementary Files}

This is a list of supplementary files associated with this preprint. Click to download.

- Additionalfile6DESeq2resDEsonly.xlsx

- Additionalfile5DESeq2resPC.TQ.xIsx

- Additionalfile4DESeq2resTC.TQ.xIsx

- Additionalfile3DESeq2resTC.PC.xlsx

- Additionalfile2Counts.xlsx

- Additionalfile1.docx 